40th "Jaszowiec" International School and Conference on the Physics of Semiconductors, Krynica-Zdrój 2011

\title{
Intermediate Band Formation and Intraband Absorption for Electrons in an Inhomogeneous Chain of Quantum Dots
}

\author{
I. BRAGAR AND P. MACHNIKOWSKI*
}

Institute of Physics, Wrocław University of Technology, Wybrzeże Wyspiańskiego 27, 50-370 Wrocław, Poland

\begin{abstract}
We study the electron states of a chain of non-identical, vertically stacked quantum dots. We discuss how the pseudo-band formed of the ground states confined in the quantum dots disintegrates upon increasing the inhomogeneity of the electron energies and analyze the impact of localization on the intraband absorption from the pseudo-band to extended (bulk) states. We describe also the dependence of the intraband absorption spectrum on the quantum dot size.
\end{abstract}

PACS: 73.21.La, 78.67.Hc, 78.30.Fs

\section{Introduction}

It has been proposed [1] that inserting a chain of vertically stacked quantum dots (QDs) in the intrinsic region of a $p-i-n$ junction solar cell could increase the photocurrent due to absorption of low energy (below bandgap) photons associated with intraband transitions between the confined states in the QD chain and the bulk continuum. While this idea has been gaining growing experimental support in the recent years [2-5], it still seems unclear whether the underlying physics corresponds to the intermediate band concept [6] as the band formation in a quantum dot chain may be strongly suppressed by the energy inhomogeneity. While the delocalized nature of the intermediate states (band formation) may not be essential for the basic idea of providing the possibility for sequential sub-bandgap absorption, it may affect the rates for the interband absorption process which will affect the efficiency of the device.

The theoretical modeling of the electron states and optical absorption in chains and arrays of QDs has so far been limited to infinite, periodic structures (superlattices) [7-11]. However, actual QD chains are always finite and usually comprise several to a few tens of stacked QDs. Even more important is the unavoidable inhomogeneity of the chain which will obviously modify the essential properties of the carrier eigenstates.

In this paper, we study the electron states of a chain of non-identical, vertically stacked quantum dots (QDs). We use a simple model of a tunnel-coupled chain of dots containing one electron, which allows us to investigate the properties of a finite, inhomogeneous system. We study how the pseudo-band formed of the ground states confined in the QDs disintegrates upon increasing the inhomogeneity of the electron energies and discuss the impact of localization on the intraband absorption from the pseudo-band to extended (bulk) states. We analyze also the dependence of the intraband absorption spectrum on the QD size.

* corresponding author; e-mail: Pawel.Machnikowski@pwr.wroc.pl
The paper is organized as follows. In Sect. 2, we describe the model of the system. Next, in Sect. 3, we characterize the eigenstates of the system and study the intraband absorption. Finally, Sect. 4 concludes the paper.

\section{Model}

We first consider single-electron states in a chain of QDs stacked along the growth direction $(z)$. Restricting the model to the ground electron state in each dot and assuming the electron spin to be fixed, we can write the Hamiltonian of the system in the form

$$
H=\sum_{n=1}^{N} \epsilon_{n}|n\rangle\langle n|+t \sum_{n=1}^{N-1}(|n\rangle\langle n+1|+\text { H.c. }),
$$

where $|n\rangle$ denotes the electron state in the $n$-th dot with the wave function $\psi_{n}(\boldsymbol{r}), \epsilon_{n}$ is the corresponding energy, $t$ is the tunnel coupling and we assume the overlap between the wave functions localized in different dots to be negligible, so that $\left\langle n \mid n^{\prime}\right\rangle=\delta_{n n^{\prime}}$. The usual inhomogeneity of the QD stack is taken into account by choosing the energies $\epsilon_{n}$ from the Gaussian distribution with the mean $\bar{E}$ and variance $\sigma^{2}$. The Hamiltonian (1) is diagonalized to yield the single-electron states $|\nu\rangle$ with the wave functions $\Psi_{\nu}(\boldsymbol{r})=\sum_{n} c_{\nu, n} \psi_{n}(\boldsymbol{r})$ and energies $E_{\nu}$, $\nu=1, \ldots, N$.

Next, we calculate the absorption coefficient for intraband dipole transitions from the initial state $\nu$ to the continuum of bulk states, which is proportional to

$$
\begin{aligned}
& \alpha(\omega) \sim \sum_{\boldsymbol{k}}\left|\hat{\mathcal{E}} \cdot \int \mathrm{d}^{3} r \Psi_{\boldsymbol{k}}^{*}(\boldsymbol{r}) \boldsymbol{r} \Psi_{\nu}(\boldsymbol{r})\right|^{2} \\
& \quad \times \delta\left(\hbar \omega+E_{\nu}-E_{\boldsymbol{k}}\right),
\end{aligned}
$$

where $\hat{\mathcal{E}}$ is the polarization of the incident light, $\Psi_{\boldsymbol{k}}(\boldsymbol{r})$ is the wave function of the final bulk state and $E_{\boldsymbol{k}}$ is its energy.

We assume that the wave functions for the confined states have the form

$$
\psi_{n}(\boldsymbol{r})=\frac{1}{\pi^{3 / 4} l l_{z}^{1 / 2}} \exp \left(-\frac{1}{2}\left(\frac{x^{2}+y^{2}}{l^{2}}+\frac{\left(z-z_{n}\right)^{2}}{l_{z}^{2}}\right)\right),
$$


where $z_{n}=n D$ is the position of the $n$-th dot ( $D$ is the distance between the dots) and $l, l_{z}$ are the extensions of the wave function in the $x y$ plane and along $z$, respectively.

For the bulk electron states, we assume the simplest approximate model of plane waves orthogonalized to the localized states,

$$
\Psi_{\boldsymbol{k}}(\boldsymbol{r})=N_{\boldsymbol{k}}\left[\frac{1}{\sqrt{V}} \mathrm{e}^{\mathrm{i} \boldsymbol{k} \boldsymbol{r}}-\sum_{n} \gamma_{\boldsymbol{k} n} \psi_{n}(\boldsymbol{r})\right],
$$

where $N_{k}$ is the appropriate normalization constant, $V$ is the normalization volume and the orthogonalization coefficients are given by

$$
\gamma_{\boldsymbol{k} n}=\frac{1}{\sqrt{V}} \int \mathrm{d}^{3} r \psi_{n}^{*}(\boldsymbol{r}) \mathrm{e}^{\mathrm{i} \boldsymbol{k} \boldsymbol{r}} .
$$

The corresponding energies are $E_{\boldsymbol{k}}=\hbar^{2} k^{2} /\left(2 m^{*}\right)$, where $m^{*}$ is the effective electron mass.

In the calculations presented in the next section, we consider a chain of 10 QDs with the inter-dot distance $D=9 \mathrm{~nm}$. Based on our earlier $\boldsymbol{k} \cdot \boldsymbol{p}$ calculations [12], we set the average energy of the confined states $\bar{E}=$ $-200 \mathrm{meV}$, and the tunnel coupling $t=-4 \mathrm{meV}$. The effective mass is $m^{*}=0.067 m_{0}$, where $m_{0}$ is the free electron mass. Each result for a random (disordered) chain has been calculated for a single realization of the system parameters.

\section{Results}

In Fig. 1, we show the occupation probabilities $P_{\nu}(n)=$ $|\langle n \mid \nu\rangle|^{2}$ for a uniform chain (a) and for two inhomogeneous chains $(b, c)$ with different standard deviations of the confinement energies $\epsilon_{n}$. For a chain of identical dots, the probabilities follow the known analytical result $P_{\nu}(n)=[1 /(N+1)] \sin ^{2}(\pi \nu n /(N+1))$. Thus, an intermediate quasi-band of delocalized QD states is formed. As expected, with increasing inhomogeneity the states tend to localize on individual dots and the intermediate band disintegrates into localized states.

The localization of the electron states considerably affects the intraband absorption from the QD states to the bulk continuum. As can be seen in Fig. 2 (red solid line), the absorption probability from the ground state of the chain has the form of a series of peaks and differs considerably from that characteristic of a single QD (black dotted line). This results from the interference effect which leads to preferred transitions to states with $k_{z}=2 \pi q / D$, where $q$ is an integer. A maximum appears around the photon energies

$$
\hbar \omega_{q}=\left|E_{1}\right|+\frac{2 \hbar^{2} \pi^{2} q^{2}}{m^{*} D^{2}}, \quad q=0,1, \ldots,
$$

where new propagation directions of the final plane wave state become allowed by energy conservation. This interference obviously requires that the initial state is delocalized over the whole chain. As the inhomogeneity increases the ground state localizes and the absorption becomes more and more similar to the single dot case, apart
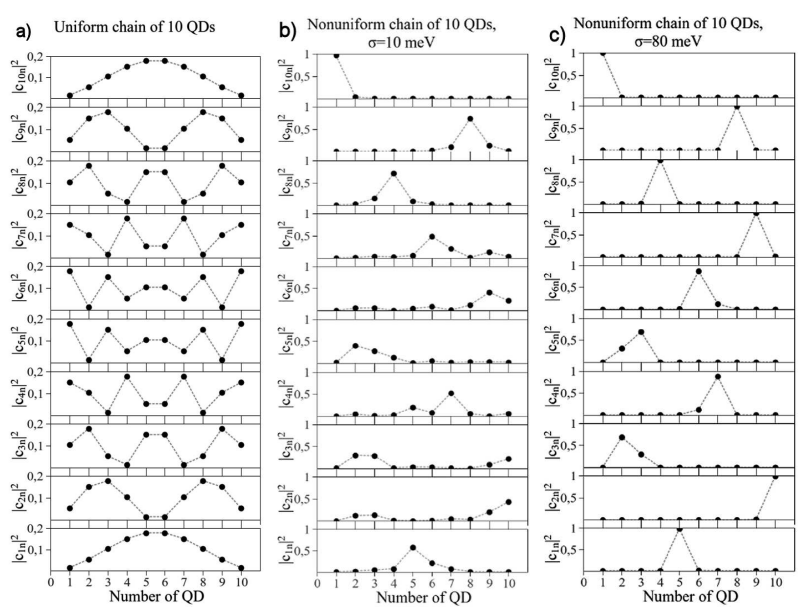

Fig. 1. The occupation probabilities for the electron eigenstates in a chain of 10 identical dots (a) and in chains with two different degrees of inhomogeneity $(\mathrm{b}, \mathrm{c})$.

from a shift, which is a trivial consequence of shifting the ground state down from the average energy $\bar{E}$ (dashed and dash-dotted lines in Fig. 2). It is quite remarkable, however, that the characteristic form of the absorption coefficient survives even under relatively strong disorder, with the standard deviation of the energies larger than the tunnel coupling constant.

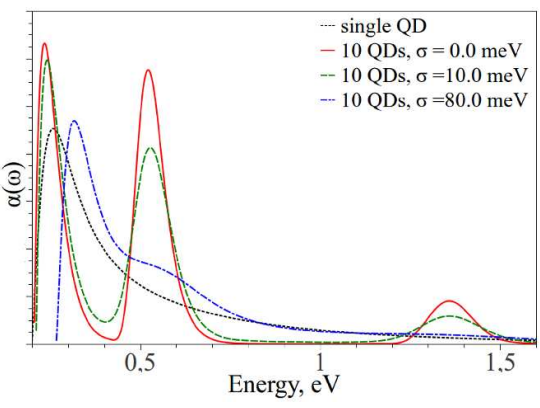

Fig. 2. The intraband absorption spectrum for a system initially in the ground state $(\nu=0)$ as a function of the photon energy $\hbar \omega$ for a single dot and for QD chains with different degrees of inhomogeneity (disorder), described by the standard deviation $\sigma$.

The form of the absorption spectrum depends on the lateral size $l$ of the confined states in QDs, as shown in Fig. 3. This is related to the increasing volume of allowed final states in the reciprocal space as the dot size becomes smaller. Apart from the very strong increase of the magnitude of the absorption in the whole frequency range for smaller dots, decreasing the QD size leads to appearance of non-zero absorption in the gaps between the absorption peaks, where the absorption is almost null for larger dots.

The dependence of the absorption spectrum on the dot height (the confinement size in the growth direction, $l_{z}$ ) 


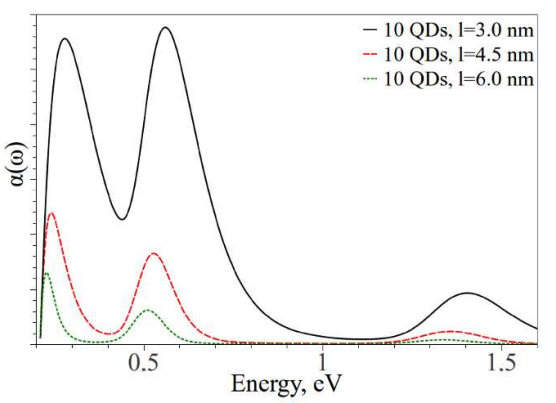

Fig. 3. The dependence of the intraband absorption spectrum on the lateral QD confinement size for an inhomogeneous chain with $\sigma=10 \mathrm{meV}$ initially in the ground state.

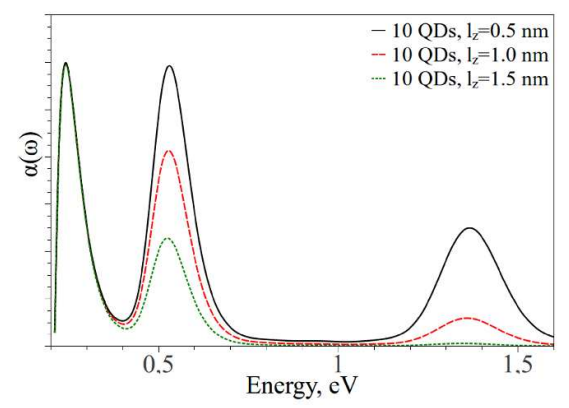

Fig. 4. The dependence of the intraband absorption spectrum on the QD confinement size in the growth direction for an inhomogeneous chain with $\sigma=10 \mathrm{meV}$ initially in the ground state.

is shown in Fig. 4. While changing the QD height affects the first peak very weakly, the dependence becomes important for higher peaks that disappear as $l_{z}$ becomes larger. This results from the fact that the higher peaks are built upon the tail of the single dot absorption (cf. Fig. 2), which is due to transitions to large momentum states propagating in the strongest confinement direction. When the single dot states become more weakly localized in this direction the momentum selection rules restrict these transitions and the tail vanishes, which also leads to disappearance of the higher absorption peaks.

\section{Conclusions}

We have studied the properties of electron states in a finite, inhomogeneous chain of quantum dots and the intraband absorption spectrum related to transitions from the lowest confined state in the QD chain to the bulk continuum states. We have shown that even for a relatively short chain of 10 dots, the intraband absorption spectrum is dominated by interference effects and differs considerably from a single dot spectrum. This effect is remarkably stable against the random variation of the energy levels in the individual dots and persists even when the standard deviation of the latter is a few times larger than the magnitude of the tunnel coupling. For dots with typical confinement sizes, the spectrum has the form of broad peaks separated by gaps where the system shows almost no absorption. The gaps can be closed by reducing the lateral dot size. On the other hand, increasing the size in the growth direction considerably reduces the magnitude of absorption peaks at higher frequencies.

\section{Acknowledgments}

This work was supported by the Foundation for Polish Science under the TEAM programme, co-financed by the European Regional Development Fund.

\section{References}

[1] V. Aroutiounian, S. Petrosyan, A. Khachatryan, K. Tourian, J. Appl. Phys. 89, 2268 (2001).

[2] R. Oshima, A. Takata, Y. Okada, Appl. Phys. Lett. 93, 083111 (2008).

[3] D. Guimard, R. Morihara, D. Bordel, K. Tanabe, Y. Wakayama, M. Nishioka, Y. Arakawa, Appl. Phys. Lett. 96, 203507 (2010).

[4] Y. Okada, R. Oshima, A. Takata, J. Appl. Phys. 106, 024306 (2009).

[5] Y. Okada, T. Morioka, K. Yoshida, R. Oshima, Y. Shoji, T. Inoue, T. Kita, J. Appl. Phys. 109, 024301 (2011).

[6] A. Luque, A. Marti, Phys. Rev. Lett. 78, 5014 (1997).

[7] Q. Deng, X. Wang, C. Yang, H. Xiao, C. Wang, H. Yin, Q. Hou, J. Li, Z. Wang, X. Hou, Physica B 406, 73 (2011).

[8] J.W. Kłos, M. Krawczyk, J. Appl. Phys. 106, 093703 (2009).

[9] J.W. Kłos, M. Krawczyk, J. Appl. Phys. 107, 043706 (2010).

[10] S. Tomić, T.S. Jones, N.M. Harrison, Appl. Phys. Lett. 93, 263105 (2008).

[11] Q. Shao, A.A. Balandin, A.I. Fedoseyev, M. Turowski, Appl. Phys. Lett. 91, 163503 (2007).

[12] K. Gawarecki, M. Pochwała, A. Grodecka-Grad, P. Machnikowski, Phys. Rev. B 81, 245312 (2010). 